\title{
Research on parallel structure's dual-frequency Fx-LMS calculation of signal filter with errors
}

\author{
ZHOU Lujun ${ }^{1, a}$, ZHOU Daoqing ${ }^{2, b}$, NIE Yongming ${ }^{3, c}$, RU Hailong ${ }^{4, d}$, HAO \\ Yong ${ }^{5, \mathrm{e}}$ \\ China Satellite Maritime Tracking and Controlling Department, Jiangyin, 214431, China

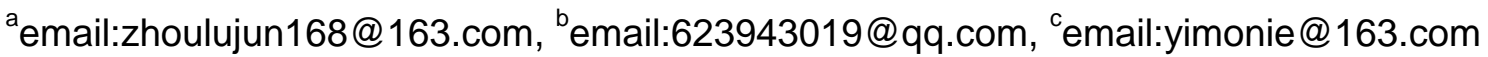

Keywords:Error signal, Parallel structure, Dual-frequency Fx-LMS calculation, Helicopter, emulation analysis.

Abstract. Parallel structure' s dual-frequency Fx-LMS calculation of signal filter with is introduced by having emulation analysis of active vibration control system in single - input and single-output way as well as four-input and two-output way while taking finite element model helicopters as research objects. Calculation emulation and analysis are made under the circumstance of time-varying secondary path, which achieved satisfied control effect.

\section{Introduction}

Active control of structural response is a high and new technology which attracts helicopter engineering industry' $\mathrm{s}$ attention at home and abroad nowadays. Modified parallel structure calculation of signal filter with errors is carried out by taking finite element model helicopter which is simplified into free-free combination beam and designing an active control system with error path off-line function in single-input and single-output way as well as four-input and two-output way for calculation emulation in the condition of dual-frequency external disturbance while traditional parallel structure Fx-LMS multi-frequency calculation is simple and efficient but unstable and inductive in spectrum. In the meanwhile, because of the actual vibration of the helicopter is more complex, the control law emulation analysis of time-varying typical secondary path is selected in order to inspect whether control ways adapt to the sophisticated environment or not, which achieved satisfied control effect.

\section{Parallel Structure's Dual-frequency Fx-LMS Calculation of Signal Processing Filter with Errors}

Single-input and single-output. As is shown in the figure, reference signals consist of two frequency components $x_{1}(n)$ and $x_{2}(n) . p(n)$ is the primary path and $s(n)$ is the error transfer function in error path. The digital model $\hat{s}(n)$ is put before reference signal and adjustment filter weight coefficient. When $w(n)$ is $p(n)$, s optimal estimation, $y(n)$ can reappear $d(n)$ better, minimizing error signal $e(n)$. Error signal frequency separation through adding digital filter can help to eliminate inductive harmonic components. 


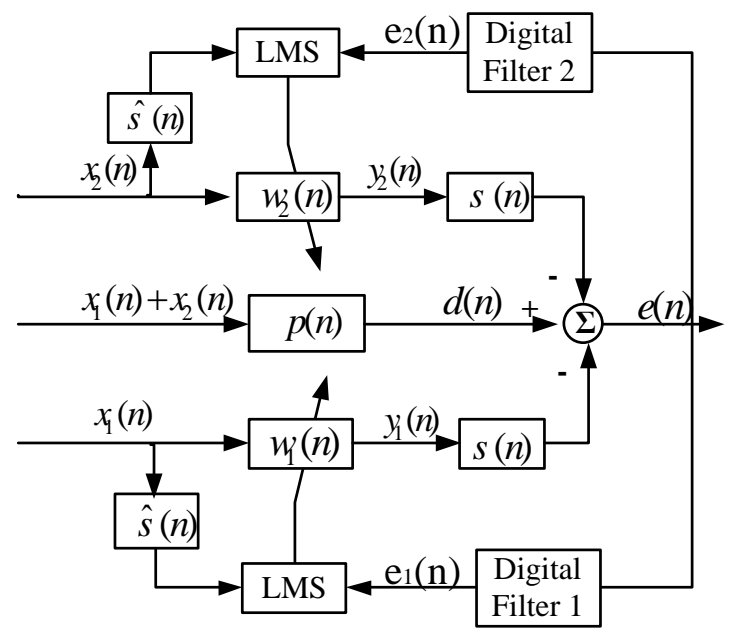

Fig1 Single-input and Single-output Dual-frequency Control System Chart

Four-input and two-output. As is shown in Figure 2, dual-frequency system of four-input and two-output has four main paths and eight error paths.

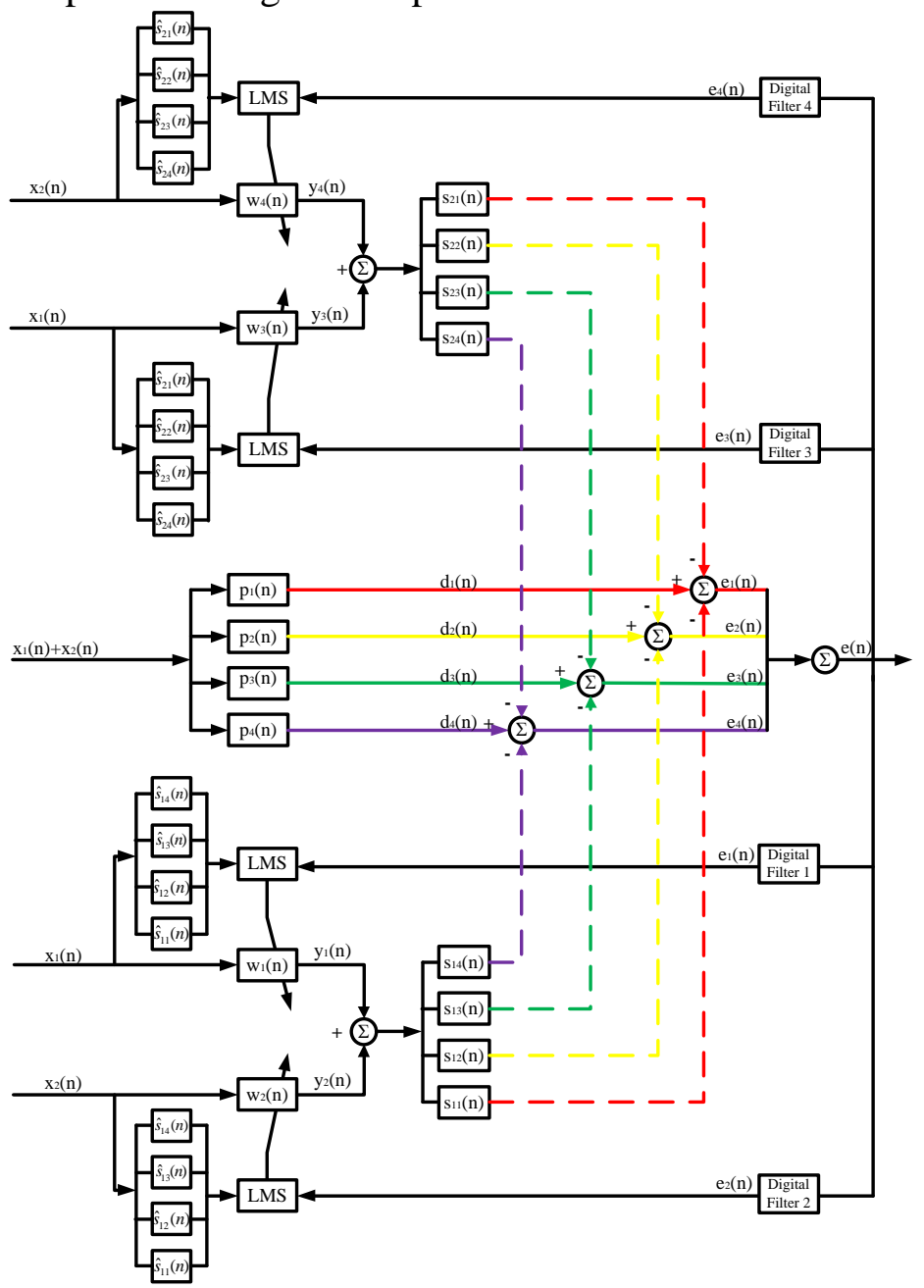

Fig 2 Four-input and Two-output Dual-frequency Control System Chart

\section{Emulation Model of Helicopter Airframe Structure}

In order to verify the feasibility and effectiveness of parallel structure dual-frequency of signal filter with errors, finite element model helicopter of free-free combination beam is selected as research object. As is shown in figure 3, this model includes mainly the airframe, tail beam, tail oblique beam, final drive support system and outer short wing. Shorter overhanging beam is added for vibration test. 


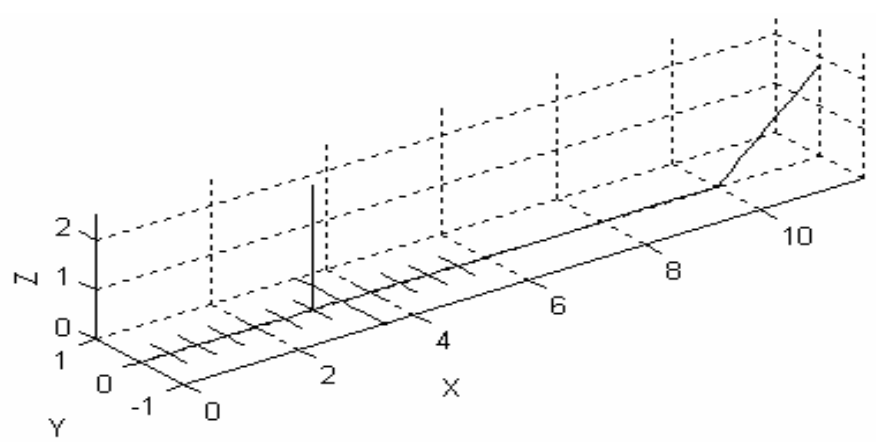

Fig. 3 Finite Element Model of Helicopter Airframe

The dimension in Figure 3 is meter. The basic parameter is: $\rho=7.8 \times 10^{3} \mathrm{~kg} / \mathrm{m}^{3} 、 \mu=0.3$ 、 $E=210 \mathrm{Gpa}$, the weight is $6.114 \times 10^{3} \mathrm{~kg}$. The accelerated speed response, regarded as error signal, in nodes of number 35, 37, 39 and 41 is used to estimate the whole performance of active control system. Figure 4 is drawing of partial enlargement of model airframe.

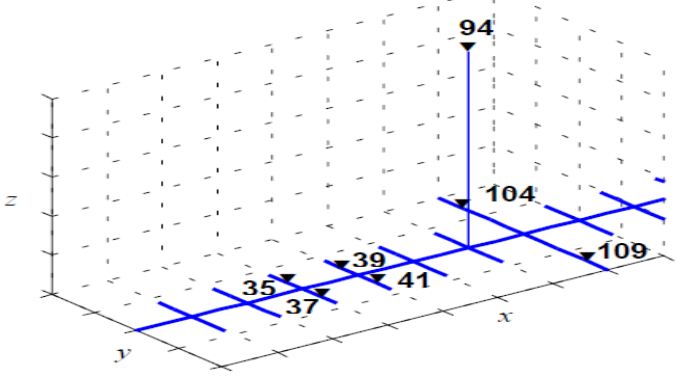

Fig 4 drawing of partial enlargement of model airframe

\section{Calculation Emulation}

\section{Single-input and Single-output Calculation Emulation}

To simulate rotor wing' s exciting force, resultant force of $1500 \mathrm{~N}$ in amplitude, $17 \mathrm{~Hz}$ in frequency, $0^{\circ}$ in phase and harmonic force with $750 \mathrm{~N}$ in amplitude, $34 \mathrm{~Hz}$ in frequency and $0^{\circ}$ in phase is introduced in node number 94 of front airframe. Controlling force is introduced in node number 104 while accelerated speed, regarded as error signal, in node number 35 is used to have emulation research. As the signals which are applied in primary path are regarded as force, the output signal from filter can be seen as controlling force of secondary path, whose unit is also N.

Harmonic Force External Disturbance Control Emulation.Sampling rate is $1000 \mathrm{~Hz}$. The order of calculation feed forward control is 64 while the initial value is 0 . Convergence coefficient are: $\mu_{17 \mathrm{~Hz}}=4 \times 10^{-5}, \quad \mu_{34 \mathrm{~Hz}}=4 \times 10^{-3}$; External disturbance stimulation will apply control after 10 seconds. The emulation result will be shown in Figure 5.

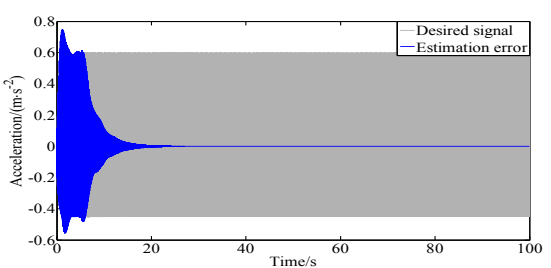

(a) Measured Points Accelerated speed response

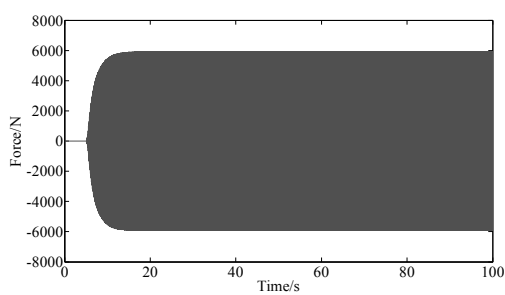

(b) Filter Output

Fig 5 Single-input and Single-output Harmonic Force External Disturbance Active Control Chart

As is shown in figure 5(a), active control has clear inhibition effect on measured points' accelerated speed: 15 seconds later than applying control, the vibration level will drop to $1 \%$ of the 
original. From Figure 5(b) can be known, controlling force increases after control and the final amplitude can reach about $5920 \mathrm{~N}$.

Control Emulation Under the Circumstance that Secondary Path Changes.Regarding signal $17 \mathrm{~Hz}+34 \mathrm{~Hz}$ as stimulation, changing secondary path amplitude frequency characteristic into 1.5 times to the original 40 seconds later and changing it into 0.5 times to the original 70 seconds later after control is converged. The convergence coefficient are $\mu_{17 \mathrm{~Hz}}=4 \times 10^{-5}, \mu_{34 \mathrm{~Hz}}=4 \times 10^{-3}$, and the emulation result is shown in Figure 6.

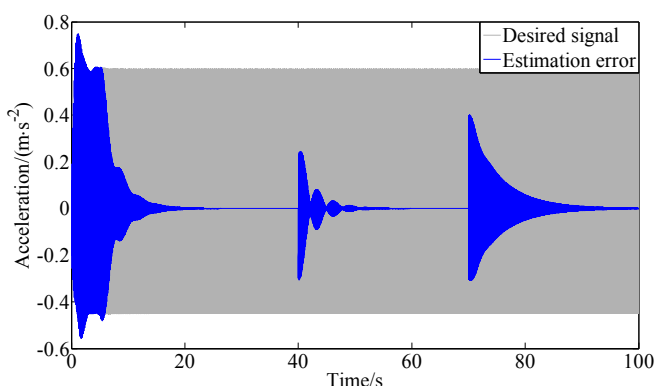

(a) Measured Points Accelerated Speed Response

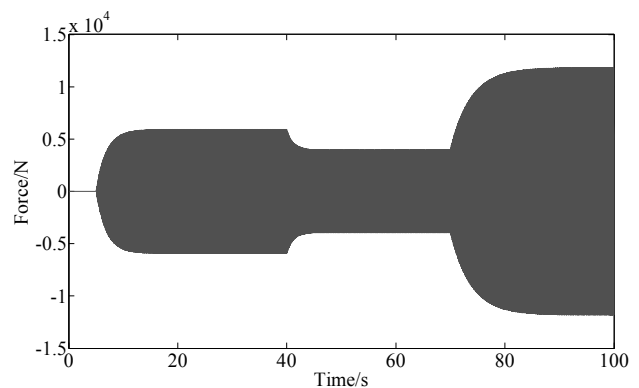

(b) Filter Output

Fig 6 Active Control Charts under the Circumstance of Changes in Secondary Path

Figure 6 shows that changes of amplitude frequency characteristic in secondary path lead to changes in accelerated speed response. Although time error estimation of both 40 seconds and 70 seconds has major fluctuation, it can be inhibited efficiently in a short time. In addition, Figure 6 also shows that when amplitude frequency characteristic in secondary path increases, filter output must reduce for keeping energy balance because the energy from external disturbance output does not change (the excitation source does not change); when amplitude frequency characteristic in secondary path decreases, filter output must increases.

In a word, parallel structure' s dual-frequency Fx-LMS calculation of signal filter with errors in single-input and single-output performs well in vibration control.

\section{Four-input and Two-output Calculation Emulation}

To simulate rotor wing' s exciting force, resultant force of $1500 \mathrm{~N}$ in amplitude, $17 \mathrm{~Hz}$ in frequency, $0^{\circ}$ in phase and harmonic force with $750 \mathrm{~N}$ in amplitude, $34 \mathrm{~Hz}$ in frequency and $0^{\circ}$ in phase is introduced in node number 94 of front airframe. Controlling force is introduced in node number 104 and node number 109 while accelerated speed response, regarded as error signal, in node number 35 , 37, 39 is used to have emulation research.

Harmonic Force External Disturbance Control Emulation.Sampling rate is $1000 \mathrm{~Hz}$. The filter order of calculation feed forward control is 64 while the initial value is 0 . The convergence coefficient are: $\mu 1_{17 \mathrm{~Hz}}=6 \times 10^{-4}, \mu 2_{17 \mathrm{~Hz}}=4 \times 10^{-4}, \mu 1_{34 \mathrm{~Hz}}=1 \times 10^{-3}, \mu 2_{34 \mathrm{~Hz}}=5 \times 10^{-3}$; Control will be applied after external disturbance stimulation begin, which is shown in Figure 7. 

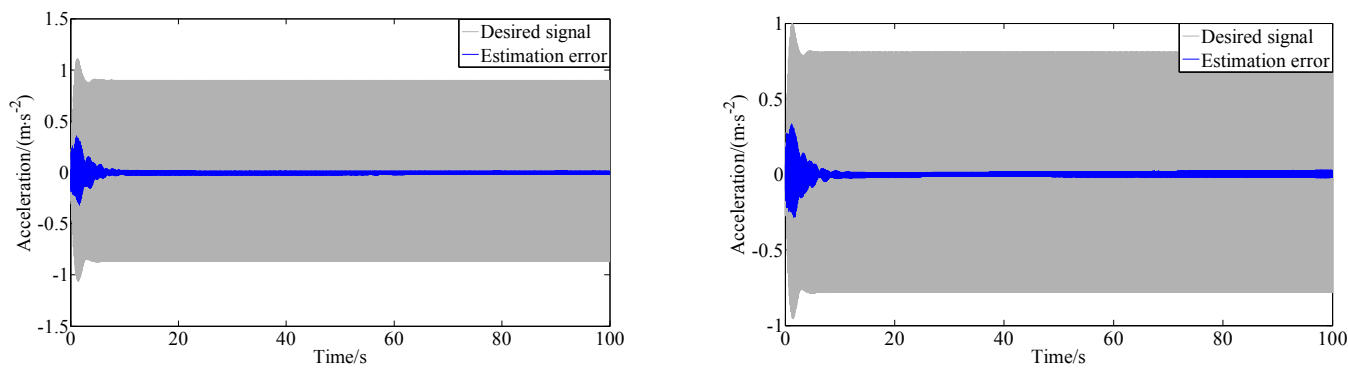

(a) Measured Point No. 39 Accelerated Speed Response (b) Measure Point No.41 Accelerated Speed Response
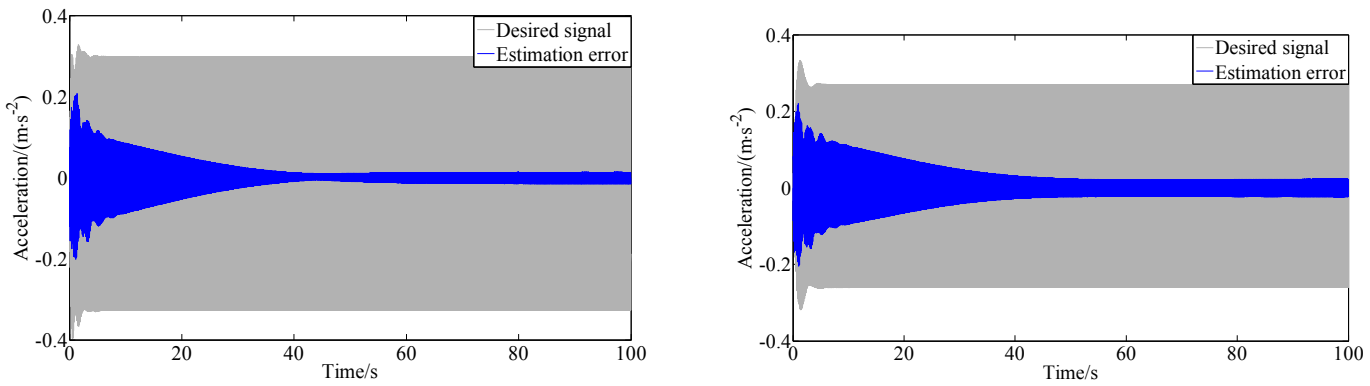

(c)Measured Point No. 35 Accelerated Speed Response (d) Measured Point No. 37 Accelerated Speed Response

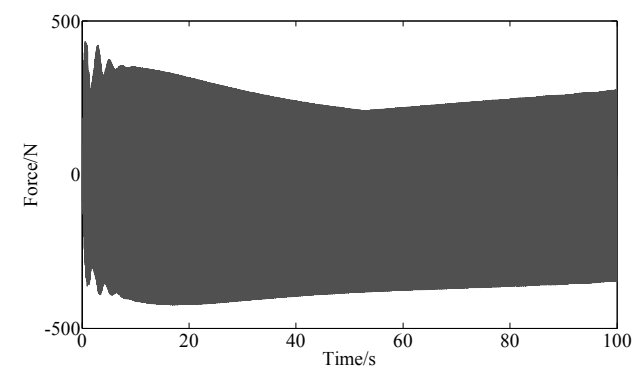

(e) Controlling Force in Node No.109

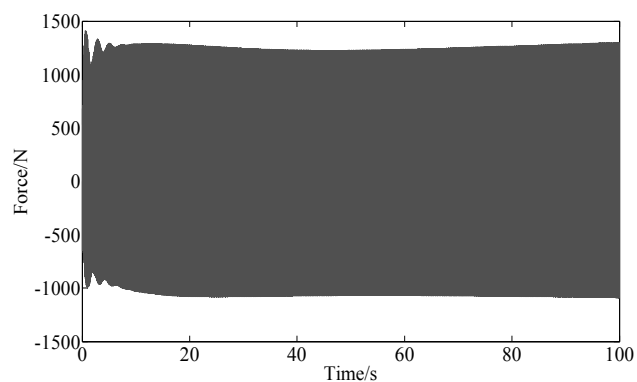

(f) Controlling Force in Node No.104

Figure 7 Active Control Chart of Four-input and Two-output Harmonic Force External Disturbance

Figure 7 shows that every measured point' s accelerated speed response is inhibited well after active control under the condition of this convergence coefficient. Measured points No.39 and 41 converges quicker that 10s later it would converge after applying control; measured points No.35 and 37 converges slower, which needs about 40s. Moreover, their damping effect are not better than previous two points mainly for the far distance from measured points No.35 and 37 to application point.

Control Emulation under the Circumstance that Secondary Path Changes.Regarding signal $17 \mathrm{~Hz}+34 \mathrm{~Hz}$ as stimulation, changing secondary path amplitude frequency characteristic into 1.5 times to the original 40 seconds later and changing it into 0.5 times to the original 70 seconds later after control is converged. The convergence coefficient are $\mu_{17 \mathrm{~Hz}}=4 \times 10^{-5}, \mu_{34 \mathrm{~Hz}}=4 \times 10^{-3}$, and the emulation result is shown in Figure 8 . 


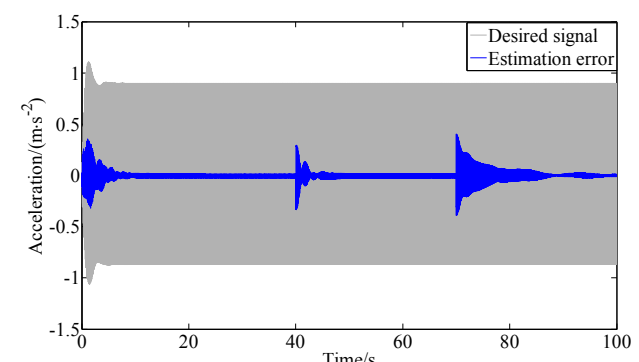

(a) Measured Point No.39 Accelerated Speed Response

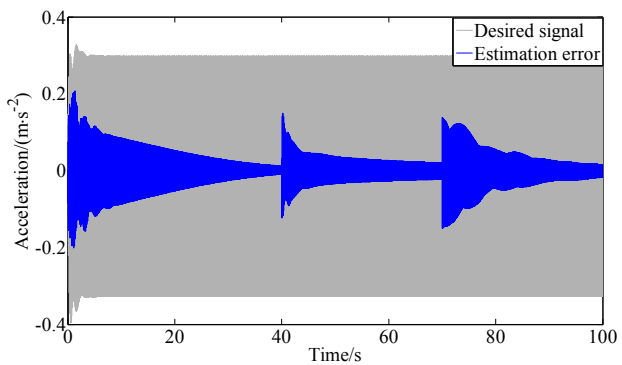

(c) Measured Point No.35 Accelerated Speed Response

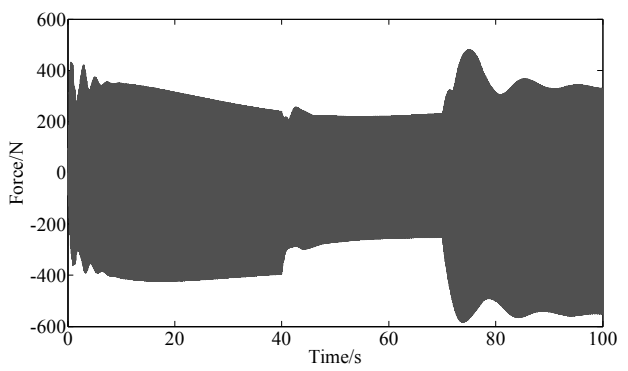

(e) Controlling Force in Node No.109

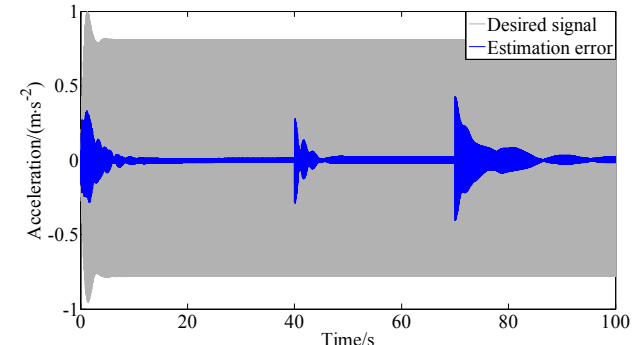

(b) Measured Point No.41Accelerated Speed Response

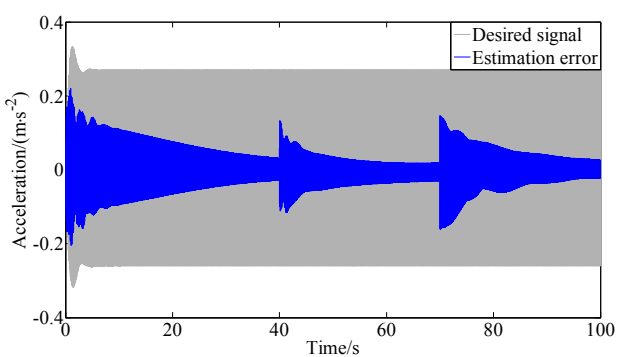

(d) Measured Point No.37 Accelerated Speed Response

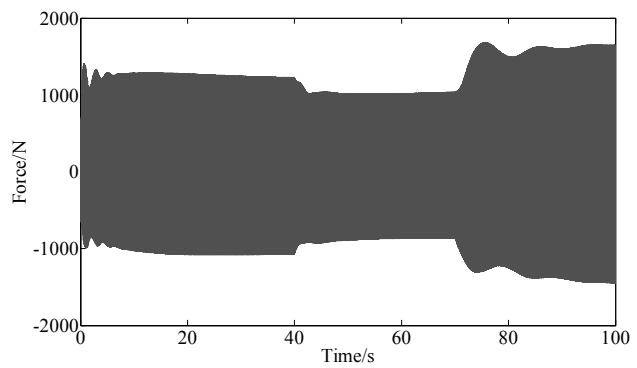

(f) Controlling Force in Node No.104

Figure 8 Active Control Charts of Changes in Secondary Path

Figure 8 shows that changes of amplitude frequency characteristic in secondary path lead to changes of accelerated speed response. Although time error estimation of both 40 seconds and 70 seconds has major fluctuation, it can be inhibited efficiently in a short time. In addition, Figure (e) and (f) also shows that when amplitude frequency characteristic in secondary path increases, filter output must reduce for keeping energy balance because the energy from external disturbance output does not change (the excitation source does not change); when amplitude frequency characteristic in secondary path decreases, filter output must increases.

\section{Summary}

Taking finite element model of simplified helicopter airframe as emulation object, vibration active control emulation research on parallel structure dual-frequency calculation of signal filter with errors in single-input and single-output way as well as four-input and two-output way is done. With the purpose of verifying the effectiveness of control calculation, the research focus on harmonic force external disturbance and time-varying in secondary path, which achieved satisfied control effect.

\section{References}

[1] Lu Y, Gu Z Q, Ling A M. Flight test of active control of structure response for helicopter. Journal of Vibration Engineering, 2012, 23(1): 24-29. (in Chinese). 
[2] Byeongil Kim, Gregory N Washington and Rajendra Singh.Control of incommensurate sinusoids using enhanced adaptive filtering algorithm based on sliding. Journal of Vibration and Control published online 8 May 2012.0(0) 1-16

[3] A. B. Puvvala, Effects of frequency separation on rate of convergence in multiple-frequency periodic noise control systems,MS thesis, Northern Illinois University, December 2004.

[4]Yang T J,G Z Q,LU M Y. Active control of helicopter structural vibration based on the error channel Online Identification. Journal of Aeronautics,2004,25(1).

[5]Yang T J,G Z Q,LU M Y. Active vibration control system based on the error channel Online Identification. Vibration and Shock,2004,23(3). 